\title{
Zero density of open paths in the Lorentz mirror model for arbitrary mirror probability
}

October 1,2018

E-mail: ata.kraemer@gmail.com E-mail: dpsanders@ciencias.unam.mx

Keywords Mirror model $\cdot$ recurrence $\cdot$ random environment

Abstract We show, incorporating results obtained from numerical simulations, that in the Lorentz mirror model, the density of open paths in any finite box tends to 0 as the box size tends to infinity, for any mirror probability.

\section{Introduction}

Lorentz gases are models that consist of particles moving in space and colliding elastically with spherical obstacles placed on the vertices of a grid, which can be periodic (see, e.g., [4, 19,20]) or aperiodic (see, e.g., [18, 17]). A similar model is the Ehrenfest wind-tree model, in which the obstacles are diamonds [3].

A version of the Ehrenfest model is to consider particles moving along the edges of a lattice, placing obstacles at the vertices that deflect particles according to a deterministic rule; such models are known as lattice Lorentz gases. Interest has focused on their diffusive properties $[15,25,11,10,9,14,24,2,8,1,7,7$, , the distribution of orbit sizes [27], topological dynamics [6] and recurrence properties [12, 5, 21, 27, 16].

One of the most-studied versions of the lattice Lorentz gas is the Lorentz mirror model [16], introduced in the physics literature by Ruijgrok and Cohen [22], where the scatterers are two-sided mirrors placed on the sites of a square lattice, $\mathbb{Z}^{2}$. These mirrors are fixed, with an orientation of 45 degrees (right) or $-45 \mathrm{de}$ grees (left), and reflect particles (or light beams) that move along the edges of the lattice. The orientation of each mirror is chosen uniformly at random, with probabilities $p_{r}$ and $p_{l}$, respectively, with $p:=p_{l}+p_{r} \leq 1$; the mirror model is thus one of the simplest realisations of deterministic dynamics in a quenched random

Departamento de Física, Facultad de Ciencias, Universidad Nacional Autónoma de México, Ciudad Universitaria, México D.F. 04510, Mexico 
environment. (There is also a flipping version of the mirror model, in which the mirrors change orientation when hit [22].) When a particle arrives at a site occupied by a mirror, it is reflected, and leaves along one of the other three edges incident on that site, following the rule given by the mirror (or absence of a mirror) there. Thus, either exactly two distinct paths touch a given mirror, or a single path touches the mirror from all directions.

For this model, it was proved that if the total density of mirrors $p=p_{r}+p_{l}$ is 1 , and both individual orientation probabilities are greater than 0 , then with probability 1 (with respect to the random environment) all trajectories are periodic, forming a closed path, [12,5].

For concentrations $0<p<1$, it was conjectured [13], based, in part, on numerical simulations [12], that the same holds for any mirror concentration $p$. However, we are not aware of any previous argument, even of a heuristic nature, to confirm or refute this conjecture. Of the few rigorous results known for $p<1$, Quas proved that if there exist open orbits, then one of the following holds: There is only one open orbit, or there are infinitely many open orbits [21]. Recently, Kozma and Sidoravicius gave a rigorous lower bound on the probability that a trajectory from the origin leaves a square box of given side length $L$.

In this letter we show, incorporating numerical results in our argument, that a weaker result holds: for any mirror density $p>0$, the density of open paths (i.e., the fraction of edges which are contained in open paths) converges to 0 in the limit $L \rightarrow \infty$.

\section{Definitions of key quantities}

We will work exclusively with finite portions of the infinite lattice, consisting of square boxes with side length $L$ : mirrors are placed on the $L^{2}$ vertices, and there are $2 L^{2}$ edges (excluding those that leave the box). The trajectory of a particle forms a path, i.e., the directed sequence of edges followed by the particle under the dynamics. In this finite version of the model, some trajectories form closed paths (periodic orbits), as in the infinite version; others escape from the finite box, and we call these open paths. Note that open paths necessarily are open on both ends, crossing from one edge of the box to another (possibly the same edge). There are thus exactly $2 L$ open paths.

The main quantities of interest in our analysis are the total lengths of all closed and open paths in the system of size $L$, i.e., the total number of edges contained in all closed paths and all open paths, respectively; we denote their means (over all realisations of the disorder in a box of size $L$ ), as functions of the mirror density $p$, by $\mathscr{C}_{L}(p)$ and $\mathscr{O}_{L}(p)$. For brevity, we denote $\mathscr{O}_{L}:=\mathscr{O}_{L}(1)$, and we similarly omit the argument for other quantities which depend on the mirror density $p$ when $p=1$. Since all edges belong either to an open path, or to a closed path, we have $\mathscr{C}_{L}(p)+\mathscr{O}_{L}(p)=2 L^{2}$ for all $p$.

The theorem of Grimmett and Bunimovich and Troubetzkoy on the infinite model states that in the limit $L \rightarrow \infty$, with probability 1 (with respect to the probability distribution [product measure] of the disorder), all trajectories close [13, 5]. This implies the weaker statement that $\mathscr{C}_{L} / 2 L^{2} \rightarrow 1$ as $L \rightarrow \infty$, and hence $\mathscr{O}_{L} / 2 L^{2} \rightarrow 0$. Our claim is that these also hold when $0<p<1$, i.e. that $\mathscr{O}_{L}(p) / 2 L^{2} \rightarrow$ 0 , for any $0<p \leq 1$, when $L \rightarrow \infty$. This implies that no phase transition occurs 


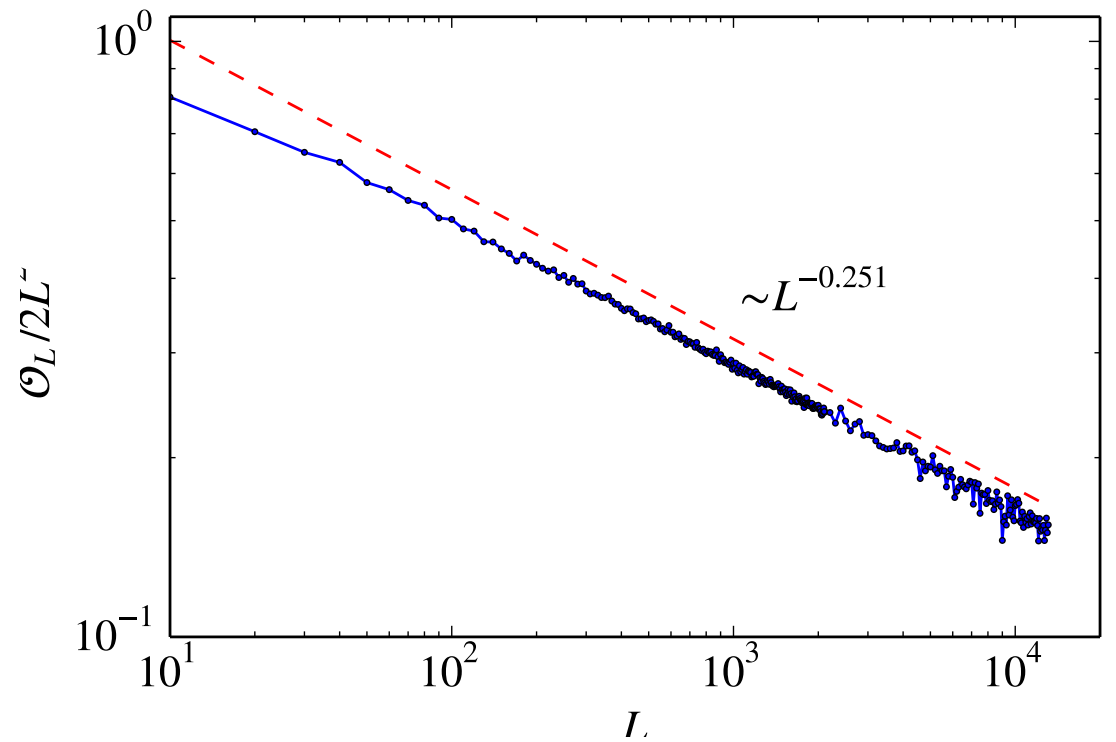

Fig. 1 Density $\mathscr{O}_{L} / 2 L^{2}$ of open paths as a function of box size $L$. The entire box of side length $L$ was simulated and each relevant quantity determined; each data point is averaged over at least 10 runs. The lines joining the points are a guide for the eye, and the dashed line shows a power-law fit to the second half of the data points.

- this would correspond to the existence of a critical probability $p_{c}$ such that a positive fraction of paths are open below $p_{c}$.

Our analysis uses the asymptotic behaviour of the density of open paths as $L \rightarrow \infty$ in a fundamental way. Figure 1 shows results of numerical simulations of this quantity. (Details of the efficient algorithms employed will be reported in a separate publication.) We find that the asymptotic behaviour is

$$
\mathscr{O}_{L} / 2 L^{2} \sim C L^{-\alpha} \quad \text { with } \quad \alpha \simeq 0.251,
$$

where $C$ is a constant. We find that $\mathscr{O}_{L} \sim L^{2-\alpha} \sim L^{1.75}$; this exponent seems to be equal to the fractal dimension $d_{f}=\frac{7}{4}$ of the trajectories [27]. To explain this, consider those open paths that traverse the box from one side of the system to the other. These belong to a percolating cluster, and are fractal with exponent 7/4 [26]. Many of the open paths will be short, of length of order $L$, being localised close to the boundaries of the square box, and thus will be dominated by the long paths that cross the system. This implies that $\mathscr{O}_{L}$ will scale as $L^{7 / 4}$; dividing by $L^{2}$ gives the exponent $\alpha=-1 / 4$.

We denote by $P_{\mathrm{c}, L}:=\mathscr{C}_{L} / 2 L^{2}$ the probability that a given $e$ dge belongs to a closed path, and $P_{\mathrm{o}, L}:=1-P_{\mathrm{c}, L}$ the probability that an edge belongs to an open path, both for mirror density $p=1$. We also denote by $P_{\mathrm{oo}, L}$ the probability that a randomly-chosen vertex separates two open paths (or a single open path with itself), and similarly for the other combinations of open and closed. 
Counting the number of open edges (those which form part of an open path) incident on each type of vertex gives the equality

$$
2 P_{\mathrm{o}, L}=P_{\mathrm{oc}, L}+2 P_{\mathrm{oo}, L} .
$$

We will study the asymptotic behaviour of the above quantities as $L \rightarrow \infty$, for which we will use the notation $A_{L} \lesssim B_{L}$ to mean that $\lim _{L \rightarrow \infty}\left(A_{L} / B_{L}\right) \leq 1$, i.e. that $A_{L}$ is asymptotically bounded above by $B_{L}$.

\section{Mirror-removal algorithm}

Our analysis is based on the idea of starting from a configuration with density $p=1$, in which each lattice site is occupied by a mirror, and removing mirrors to produce a new configuration with density $p<1$. We will analyse how the quantities defined above change during this process, and we will obtain an upper bound for the total length of open paths.

Consider the removal of a single mirror. In either the finite or infinite version of the model, one of the following possibilities occurs: (i) two distinct closed paths join together, forming a single closed path with the same total length; (ii) two distinct open paths exchange parts of their paths, maintaining the same total open length; (iii) an open path joins with a closed path, incorporating the closed path into the open path; (iv) the chosen mirror separates two parts of the same path (either open or closed). In case (iv), there are two possibilities: (a) the type and length of the path are unaffected, but the order in which edges are visited changes; or (b) the path is divided into a closed path and another path with the same type as the original path before removing the mirror (closed or open). The possible situations are illustrated in figure 2 [cases (i)-(iv)(a)] and figure 3 [case (iv)(b)].

Our algorithm proceeds by removing the required fraction of mirrors from the $p=1$ configuration (all vertices occupied by mirrors), in a certain order, in order to produce a configuration with $p<1$; any configuration with $p<1$ may be produced in this way. The algorithm is as follows:

(1) Select, uniformly at random, the sites in the $L \times L$ box at which mirrors will be removed, each with probability $1-p$, independently of the rest; call this set $\mathscr{M}$.

(2) Remove, one by one, those mirrors in $\mathscr{M}$ that separate two distinct closed paths.

(3) Remove those mirrors in $\mathscr{M}$ that join a closed path with itself.

(4) Remove the mirrors in $\mathscr{M}$ that connect an open path with a closed path.

(5) Remove the remaining mirrors in $\mathscr{M}$, i.e., those that connect an open path with itself or with a different open path.

Note that since step (3) does not modify the total length of open paths, while step (5) can only reduce this length by producing closed paths (see figure 3, we do not need to take them into account to obtain an upper bound. 


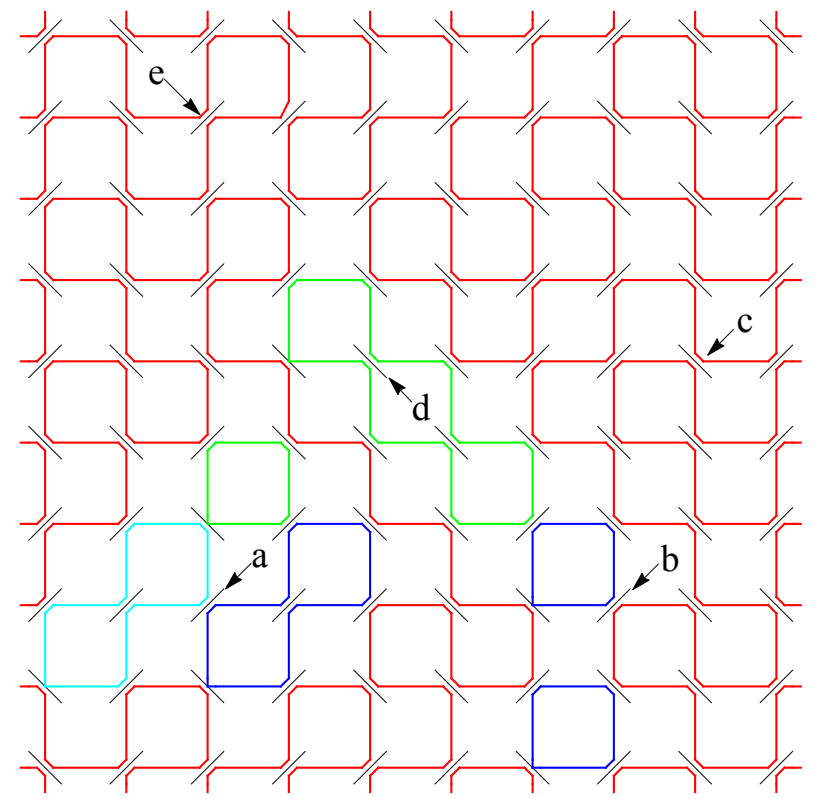

Fig. 2 Trajectories in a $10 \times 10$ box. Open paths (those that leave the box) are shown in red, and touching closed paths are shown in different colours. Labels indicate the different possible mirror types: (a) separating two distinct closed paths; (b) separating an open and a closed path; (c) separating two distinct open paths; (d) embedded in a single closed path; and (e) embedded in a single open path.

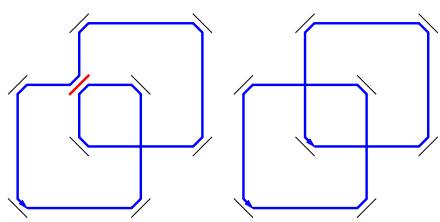

(a)
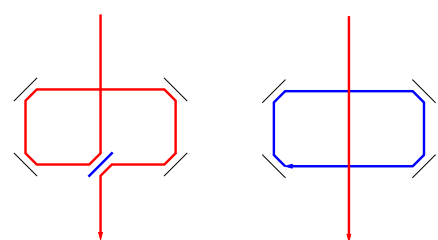

(b)

Fig. 3 Creation of a closed path after removing a mirror. Red arrows represent open paths; blue arrows represent close paths. (a) Two disjoint (but crossing) closed paths generated by removing a mirror that separates a closed path from itself. (b) A closed path crossed by an open path, generated by removing a mirror that separates an open path from itself.

\subsection{Joining distinct closed paths}

First consider step (2), in which mirrors that join two distinct closed paths are removed, creating a single closed path. Here, an order must be chosen in which to remove this subset of mirrors. When each mirror is checked, it is removed only if it currently separates two distinct closed paths. The exact configuration of closed paths obtained at the end of this step thus depends on the order in which these mirrors are removed. Nonetheless, all the different configurations obtained, by removing these mirrors in any order, have the same number of closed paths, 
which we denote by $N_{L}(p)$, the same length $\mathscr{C}_{L}$ (since the total length of closed paths has not changed in the process), and the same number of mirrors removed, which we denote by $r_{L}(p)$.

Each of the mirrors removed in this step reduces the number of closed paths by 1 . Thus

$$
N_{L}(p)=N_{L}-r_{L}(p)
$$

where $N_{L}:=N_{L}(1)$ is the number of closed paths when $p=1$. Note that $r_{L}(p)$ is bounded above by $N_{L}-1$.

We will argue that $N_{L}(p) \gtrsim g(p) N_{L}$, where $g(p)$ is a positive function of $p$ and $N_{L}:=N_{L}(1)$ is the number of closed paths when $p=1$. Consider the closed paths with exactly four edges, which we call "square paths", and in particular those square paths that are not in contact with another square path. There is a positive probability that a given bond belongs to one of these paths; call this probability $\rho$. Since these square paths have four vertices, and none of them belongs to another square path, there are exactly 4 independent mirrors for each of these paths. Thus, the probability that one of the mirrors that belong to a given one of these closed paths is removed is $p^{4}$, and hence, the number of these closed paths after step (2) is bounded below by $p^{4} \rho L^{2} / 2$, so that $N_{L}(p) \gtrsim p^{4} \rho L^{2} / 2$. Since $N_{L} / L^{2} \sim K \simeq 0.1$ (as measured numerically), for any $p>0$ we have

$$
N_{L}(p) \gtrsim \frac{p^{4} \rho K}{2} N_{L}=: g(p) N_{L}
$$

with $g(p):=\frac{p^{4} \rho K}{2}>0$.

\subsection{Joining closed and open paths}

In step (4), we remove those mirrors that connect an open path with a closed path. In this case, the closed path is amalgamated into the open path, so that the total open length grows by the length of this closed path.

To estimate this increase, we use that the expected increase in the total open length is the mean length of those closed paths that touch at least one open path; this mean length we denote by $\ell_{\mathrm{co}, L}$. To use this, we relate it to the mean length of all closed paths, $\ell_{\mathrm{c}, L}:=\mathscr{C}_{L} / N_{L}(p) \sim \mathscr{C}_{L} /\left[N_{L} g(p)\right]$. (Note that the closed paths referred to are those after the removal step (2).) Figure 4 shows that for $p=1$, we have

$$
\frac{\ell_{\mathrm{c}, L}}{\ell_{\mathrm{co}, L}} \sim C^{\prime} L^{-\beta} \quad \text { with } \quad \beta \simeq 0.0924,
$$

and a constant $C^{\prime}$. (The efficient algorithm used for this calculation will be described in a separate publication.)

It is an open question whether this exponent $\beta$ is related with known quantities in percolation, as occurs with the exponent $\alpha$. One possibility is the fractal dimension of the 2D percolation cluster, $d_{f}=91 / 48$ [23]. In figure 5]we plot separately $\ell_{\mathrm{c}, L}$ and $\ell_{\mathrm{co}, L}$ as functions of $L$. Although neither of these two quantities appears to follow a power law, their ratio does. For sufficiently large values of $L$, we have that $\ell_{\mathrm{c}, L}(L)$ may be estimated as $\ell_{\mathrm{c}, L} \sim A\left(1-L^{-1 / 4}\right)$, with $A \simeq 20$. This result is 


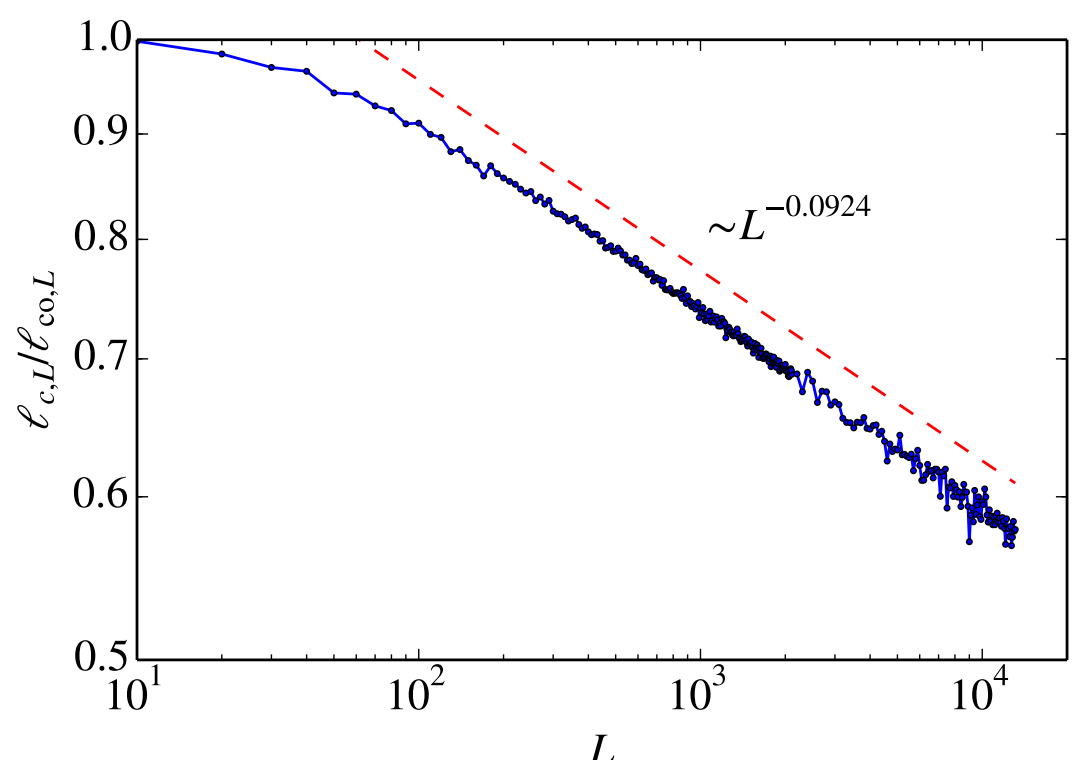

Fig. 4 Ratio $\ell_{\mathrm{c}, L} / \ell_{\mathrm{co}, L}$ of mean closed path length to mean length of those closed paths that touch open ones (double logarithmic scale). Each data point is averaged over at least 10 runs.

obtained by using that $\mathscr{O}_{L} / 2 L^{2} \sim L^{-1 / 4}$ (see figure 1 , the definition $\ell_{\mathrm{c}, L}:=\mathscr{C}_{L} / N_{L}$ for $p=1$, and the fact that $N_{L} \sim K L^{2}$, where $K$ is a constant.

For other values of $p$, we will assume that $\frac{\ell_{c, L}(p)}{\ell_{\mathrm{co}, L}(p)} \sim L^{-\beta(p)}$. We wish to show that $\beta(p) \leq \beta$ for all $0<p \leq 1$. We have

$$
\frac{\ell_{\mathrm{c}, L}(p)}{\ell_{\mathrm{co}, L}(p)}=\frac{\mathscr{C}_{L} / N_{L}(p)}{\mathscr{C}_{\mathrm{co}, L}(p) / N_{\mathrm{co}, L}(p)}=\frac{\left[\mathscr{C}_{\mathrm{co}, L}(p)+\mathscr{C}_{r, L}(p)\right] / N_{L}(p)}{\mathscr{C}_{\mathrm{co}, L}(p) / N_{\mathrm{co}, L}(p)} \sim L^{-\beta(p)},
$$

where $\mathscr{C}_{\mathrm{co}, L}(p)$ is the length of the closed paths that touch an open path, $\mathscr{C}_{r, L}(p)$ is the length of the other closed paths and $N_{\mathrm{co}, L}(p)$ is the number of closed paths touching open paths. After reducing equation $(6)$, we obtain

$$
\frac{\ell_{\mathrm{c}, L}(p)}{\ell_{\mathrm{co}, L}(p)}=\left[1+\frac{\mathscr{C}_{r, L}(p)}{\mathscr{C}_{\mathrm{co}, L}(p)}\right] \frac{N_{\mathrm{co}, L}(p)}{N_{L}(p)} \sim L^{-\beta(p)}
$$

Suppose that the worst case occurs: $\frac{\mathscr{C}_{r, L}(p)}{\mathscr{C}_{\mathrm{co}, L}(p)} \sim 0$, so that $\frac{N_{\mathrm{co}, L}(p)}{N_{L}(p)} \sim L^{-\beta(p)}$. Using similar arguments to those used above to show $N_{L}(p) \sim g(p) N_{L}$, we have $N_{\mathrm{co}, L}(p) \gtrsim g_{1}(p) N_{\mathrm{co}, L}$, where $g_{1}(p)$ is a positive function of $p$. Thus, $\frac{N_{\mathrm{co}, L}(p)}{N_{L}(p)} \gtrsim$ $f_{1}(p) \frac{N_{\mathrm{co}, L}}{N_{L}} \sim f_{1}(p) L^{-\beta}$, where $f_{1}(p)$ is again a positive function of $p$. Thus, $0 \leq$ $\beta(p) \leq \beta$.

The mean number of mirrors removed that join an open and a closed path is bounded above by $(1-p) 2 L^{2} P_{\mathrm{oc}, L}$. (Note that some mirrors join closed paths with 


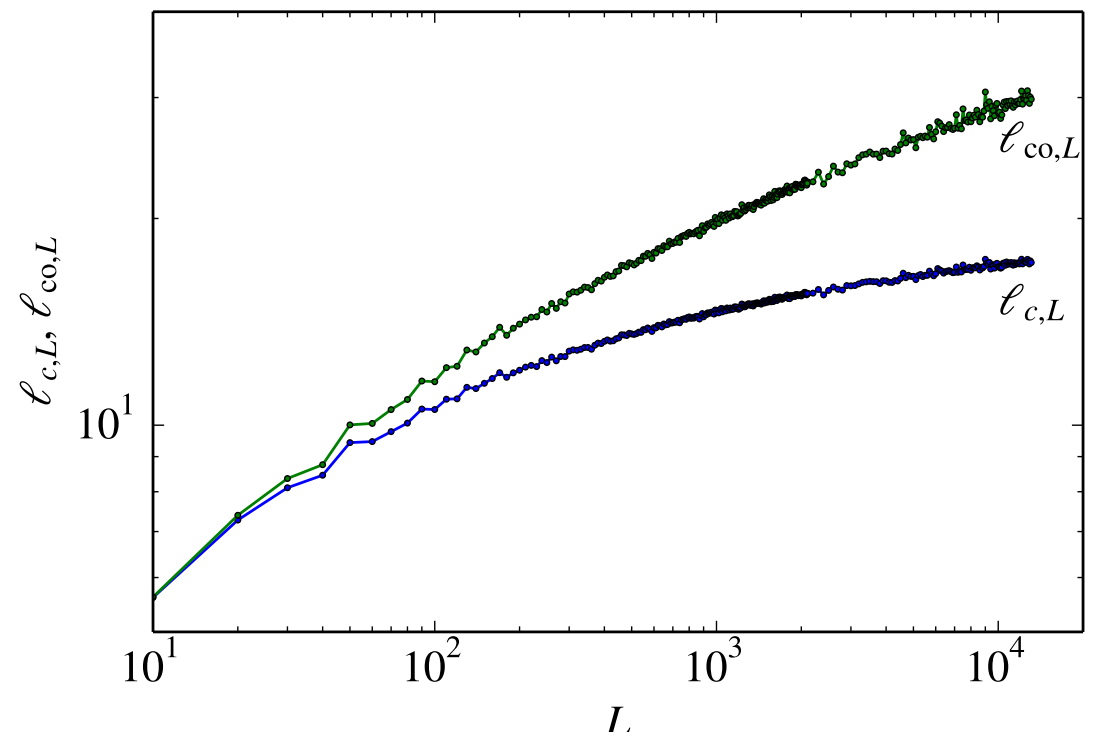

Fig. 5 Mean closed path length, $\ell_{c, L}$, and mean length of closed paths that touch open ones, $\ell_{\mathrm{co}, L}$ as a function of $L$ (double logarithmic scale).

themselves.) Thus we have

$$
\mathscr{O}_{L}(p) \lesssim \mathscr{O}_{L}+(1-p) 2 L^{2} P_{\mathrm{oc}, L} \ell_{\mathrm{co}, L}(p) .
$$

(Here we have assumed that each joined closed path may be treated independently of the others, so that the total length increase is just given by the mean length increase times the number of such closed paths.)

Dividing equation (8) by $2 L^{2}$ and using $\mathscr{O}_{L} / 2 L^{2} \lesssim C L^{-\alpha}$, we have

$$
\frac{\mathscr{O}_{L}(p)}{2 L^{2}} \lesssim C L^{-\alpha}+(1-p) P_{\mathrm{oc}, L} \ell_{\mathrm{co}, L}(p) .
$$

Now, using $\ell_{\mathrm{co}, L}(p) \sim C^{\prime} L^{\beta(p)} \ell_{\mathrm{c}, L} \lesssim C^{\prime} L^{\beta} \ell_{\mathrm{c}, L}$ and $\ell_{\mathrm{c}, L}=\mathscr{C}_{L} / N_{L}(p)$ and the bound $N_{L}(p) \gtrsim g(p) N_{L}$, we obtain

$$
\frac{\mathscr{O}_{L}(p)}{2 L^{2}} \lesssim C L^{-\alpha}+\frac{(1-p)}{g(p)} P_{\mathrm{oc}, L} \frac{\mathscr{C}_{L}}{N_{L}} C^{\prime} L^{\beta} .
$$

Now, since $P_{\mathrm{oc}, L} \lesssim 2 P_{\mathrm{o}, L}$, we have

$$
P_{\mathrm{oc}, L} \frac{\mathscr{C}_{L}}{N_{L}} \lesssim 2 P_{\mathrm{o}, L} \frac{\mathscr{C}_{L}}{N_{L}}=2 \frac{\mathscr{O}_{L}}{N_{L}} \frac{\mathscr{C}_{L}}{2 L^{2}} \lesssim 2 \frac{\mathscr{O}_{L}}{N_{L}},
$$

since $P_{\mathrm{o}, L}=\mathscr{O}_{L} / 2 L^{2}$ and $\mathscr{C}_{L} / 2 L^{2} \rightarrow 1$. Thus, equation 10 gives

$$
\frac{\mathscr{O}_{L}(p)}{2 L^{2}} \lesssim C L^{-\alpha}+\frac{2(1-p)}{g(p)} C^{\prime} \frac{\mathscr{O}_{L}}{N_{L}} L^{\beta} .
$$


Recalling that $N_{L} \gtrsim K L^{2}$, we finally have

$$
\frac{\mathscr{O}_{L}(p)}{2 L^{2}} \lesssim C L^{-\alpha}+\frac{2(1-p) C^{\prime}}{K g(p)} \frac{\mathscr{O}_{L}}{L^{2}} L^{\beta}
$$

and hence

$$
\frac{\mathscr{O}_{L}(p)}{2 L^{2}} \lesssim C L^{-\alpha}+\frac{2(1-p) C^{\prime}}{K g(p)} L^{\beta-\alpha} .
$$

Since our numerical results show that $\beta<\alpha$, we conclude that $\frac{\mathscr{O}_{L}(p)}{2 L^{2}} \sim 0$, that is, the density of open paths tends to 0 , or equivalently the density of closed paths tends to 1 , as the box size goes to infinity, for any mirror density $p$.

\section{Conclusions}

In this paper, we have shown, using input from numerical calculations, that in the limit of infinite system size the density of open paths in the Lorentz mirror model tends to 0 for any mirror probability $0<p \leq 1$. This is a weaker form of the conjecture that with probability 1 all trajectories close.

Our argument reduces the problem to the analysis of the two exponents $\alpha$ and $\beta$, which we calculated numerically via efficient simulations. Clearly, it is important to calculate $\beta$ analytically. Furthermore, our analysis has dealt only with mean quantities; it is also necessary to study the probability distributions of the corresponding random variables.

The authors thank L. Bunimovich, E. Crane, T. LaGatta and R. Díaz for useful discussions and comments on earlier drafts, and an anonymous referee for useful suggestions which improved the manuscript. Financial support from CONACYT grant CB-101246 and DGAPA-UNAM PAPIIT grants IN116212 and IN117214 is acknowledged.

\section{References}

1. Acedo, L., Santos, A.: Diffusion in lattice Lorentz gases with mixtures of point scatteres. Phys. Rev. E 50, 4577 (1994)

2. van Beijeren, H., Ernst, M.H.: Diffusion in Lorentz lattice gas automata with backscattering J. Stat. Phys. 70, 793 (1993)

3. Bianca, C.: On the existence of periodic orbits in nonequilibrium Ehrenfest gas. In: International Mathematical Forum, vol. 7, pp. 221-232 (2012)

4. Bruin, C.: A computer experiment on diffusion in the Lorentz gas. Physica 72(2), 261-286 (1974)

5. Bunimovich, L.A., Troubetzkoy, S.E.: Recurrence properties of Lorentz lattice gas cellular automata. J. Stat. Phys. 67(1), 289-302 (1992)

6. Bunimovich, L.A., Troubetzkoy, S.E.: Topological dynamics of flipping Lorentz lattice gas models. J. Stat. Phys. 72, 297 (1993)

7. Cohen, E.: New types of diffusion in lattice gas cellular automata. In: M. Mareschal, B. Holian (eds.) Microscopic Simulations of Complex Hydrodynamic Phenomena, NATO ASI Series, vol. 292, pp. 137-152. Springer US (1992)

8. Cohen, E., Wang, F.: New results for diffusion in Lorentz lattice gas cellular automata. J. Stat. Phys. 81, 445 (1995)

9. Cohen, E., Wang, F.: Diffusion and propagation in Lorentz lattice gases. Fields Institute Communications 6, 43 (1996) 
10. Ernst, M.H., van Velzen, G.A.: Lattice Lorentz gas. J. Phys. A: Math. Gen. 22, 4611 (1989)

11. Ernst, M.H., van Velzen, G.A.: Long-time tails in lattice Lorentz gases. J. Stat. Phys. 57, 455 (1989)

12. Grimmett, G.: Percolation and disordered systems. In: Lectures on Probability Theory and Statistics, pp. 153-300. Springer (1997)

13. Grimmett, G.: Percolation, 2nd edn. Springer-Verlag, New York (1999)

14. Kong, X.P., Cohen, E.G.D.: Diffusion and propagation in triangular Lorentz lattice gas cellular automata. J. Stat. Phys. 62, 737 (1991)

15. Kong, X.P., Cohen, E.G.D.: Lorentz lattice gases, abnormal diffusion, and polymer statistics. J. Stat. Phys. 62, 1153 (1991)

16. Kozma, G., Sidoravicius, V.: Lower bound for the escape probability in the Lorentz mirror model on the lattice. arXiv:1311.7437 [math.PR] pp. 1-2 (2013)

17. Kraemer, A.S., Sanders, D.P.: Embedding quasicrystals in a periodic cell: Dynamics in quasiperiodic structures. Phys. Rev. Lett. 111(12), 5501 (2013)

18. Machta, J., Moore, S.M.: Diffusion and long-time tails in the overlapping Lorentz gas. Phys. Rev. A 32, 3164-3167 (1985)

19. Machta, J., Zwanzig, R.: Diffusion in a periodic Lorentz gas. Phys. Rev. Lett. 50, 1959-1962 (1983)

20. Moran, B., Hoover, W.G.: Diffusion in a periodic Lorentz gas. J. Stat. Phys. 48, 709-726 (1987)

21. Quas, A.N.: Infinite paths in a Lorentz lattice gas model. Prob. Theor. Rel. Fields 114(2), 229-244 (1999)

22. Ruijgrok, T.W., Cohen, E.: Deterministic lattice gas models. Phys. Lett. A 133(7), 415-418 (1988)

23. Sahimi, M.: Applications of percolation theory. Taylor and Francis (2009)

24. Wang, F., Cohen, E.: Diffusion in Lorentz lattice gas cellular automata: The honeycomb and quasi-lattices compared with the square and triangular lattices. J. Stat. Phys. 81, 467 (1995)

25. X.P. Kong, E.C.: A kinetic theorist's look at lattice gas cellular automata. Physica D 47, 9-18 (1991)

26. Ziff, R.M.: Hull-generation walks. Phys. D 38, 377-383 (1989)

27. Ziff, R.M., Kong, X., Cohen, E.: Lorentz lattice-gas and kinetic-walk model. Phys. Rev. A 44(4), 2410 (1991) 